\title{
Efficacy of Daily Cone-Beam Computed Tomography as Part of a Rescan Protocol for Large Offset to Reduce the Inter-Fractional Motion of the Prostate
}

\author{
Shinsaku Yamaguchi1, Takayuki Ohguri ${ }^{*}$, Hajime Imada ${ }^{2}$, Katsuya Yahara1, \\ Hiroyuki Narisada², Satoshi Iwasaki², Toshihiro Onoda², Yuta Ezaki², Eiji Hamada², \\ Yukunori Korogi ${ }^{1}$ \\ ${ }^{1}$ Department of Radiology, University of Occupational and Environmental Health, Kitakyushu, Japan \\ ${ }^{2}$ Department of Cancer Therapy Center, Tobata Kyoritsu Hospital, Kitakyushu, Japan \\ Email: ${ }^{*}$ ogurieye@med.uoeh-u.ac.jp
}

Received 7 July 2014; revised 6 August 2014; accepted 1 September 2014

Copyright (C) 2014 by authors and Scientific Research Publishing Inc.

This work is licensed under the Creative Commons Attribution International License (CC BY). http://creativecommons.org/licenses/by/4.0/

(c) (i) Open Access

\section{Abstract}

Purpose: To prospectively analyze the inter-fractional motion of the prostate in patients with prostate cancer treated with intensity-modulated radiation therapy (IMRT) using image-guided radiotherapy (IGRT) with daily cone-beam computed tomography (CBCT) as part of a rescan protocol for large offset, and to evaluate the efficacy of our protocol. Materials and Methods: Eligible patients were treated with the following protocol: 1) magnesium oxide and dimethylpolysiloxane were administered to ensure that patients had regular bowel movements; 2 ) the patients were instructed to have an appropriately distended bladder during the planning CT and daily irradiation; 3) the daily CBCT image was fused with the planning CT image using the prostate outline; and 4) if large offset was recognized, a rescan CBCT image was obtained after appropriate countermeasures, such as the discharge of gas and defecation, and re-registration was performed. Three shifts for the inter-fractional motion of the prostate were analyzed, in the fractions which needed the CBCT rescan; the displacement data after the final rescan were used. Results: Sixty-one patients were eligible, and a total of 2302 fractions were available for the analysis. Rescans of the CBCT for large offset were performed in $113(5 \%)$ of the 2302 fractions. After the first rescan, the large offset was resolved in $106(94 \%)$ of the 113 fractions. Excessive rectal gas was the reason for the large offset in $94(83 \%)$ of the 113 fractions. The total mean and standard deviation of the inter-fractional motion of the prostate in the AP, LR, and SI directions were $1.1 \pm 2.4,-0.1 \pm 2.3$, and $0.7 \pm 3.0 \mathrm{~mm}$, respectively. Conclusion: Large offset was recognized in $5 \%$ of all fractions. Daily

\footnotetext{
*Corresponding author.

How to cite this paper: Yamaguchi, S., Ohguri, T., Imada, H., Yahara, K., Narisada, H., Iwasaki, S., Onoda, T., Ezaki, Y., Hamada, E. and Korogi, Y. (2014) Efficacy of Daily Cone-Beam Computed Tomography as Part of a Rescan Protocol for Large Offset to Reduce the Inter-Fractional Motion of the Prostate. International Journal of Medical Physics, Clinical Engineering and Radiation Oncology, 3, 193-200. http://dx.doi.org/10.4236/ijmpcero.2014.34025
} 
CBCT with our rescan protocol could resolve the large offset, which was mainly caused by excessive rectal gas, and it may therefore be promising to reduce the inter-fractional motion of the prostate.

\title{
Keywords
}

\author{
Intensity-Modulated Radiotherapy, Prostate Cancer, Daily Cone Beam Computed Tomography, \\ Inter-Fractional Motion
}

\section{Introduction}

Intensity-modulated radiotherapy (IMRT) has proven to be a useful tool because of its ability to conform high doses of radiation to the target volume. For patients with clinically localized prostate cancer, IMRT is used to escalate the dose to the prostate while minimizing the dose to the surrounding normal tissues [1]. This requires accurate daily targeting during the entire course of external beam radiotherapy to the prostate. More accurate delivery of the prescribed dose would allow for the reduction of the safety margin around the clinical target volume (CTV). Many recent studies have focused on localizing and quantifying prostate displacement [2]-[4]. Various targeting modalities have been used, such as two-dimensional portal imaging, and fiducial marker matching to three-dimensional (3D) volumetric computed tomography (CT) and ultrasound information [5]-[7]. These findings may offer the potential for an increased dose to be safely delivered to the target with reduced acute and late toxicity rates.

Kilovoltage (KV) cone beam computed tomography (CBCT) is one of the methods used to assess and correct for the inter-fractional prostate motion. It enables direct visualization of the soft-tissue targets and organs at risk. These images are acquired using a flat panel detector by rotation of a KV X-ray source mounted on the accelerator gantry orthogonal to the primary treatment axis. Unlike conventional CT scanners, CBCT reconstructs an entire image volume from a single gantry rotation.

Rectal filling and bladder volume are the main factors affecting the prostate inter-fractional motion in patients with prostate cancer [8] [9]. Antolak et al. reported that the prostate target volume variations during a course of radiotherapy, and the CTV mobility were correlated with rectal volume changes [9]. Melian et al. showed that the anterior-posterior (AP) and superior-inferior (SI) movements in the prostate position correlated with the bladder volume, while the rectal volume correlated with the observed AP motion [8].

In this context, we hypothesized that a rescan of CBCT and re-registration after appropriate countermeasures were taken (i.e. discharge of gas, defecation and urination) could positively contribute to a reduction in the inter-fractional motion of the prostate when large offset in a fusion image, consisting of planning CT and initial CBCT images, was recognized using the prostate outline. A prospective study of the daily CBCT was designed as part of a rescan protocol during IMRT to reduce the inter-fractional motion of the prostate, and the inter-fractional motion of the prostate during this protocol was prospectively collected and analyzed. The purpose of this study was to analyze the inter-fractional motion of the prostate in the patients with prostate cancer treated with IMRT using daily CBCT with a rescan protocol for large offset, and to evaluate the efficacy of our protocol.

\section{Materials and Methods}

\subsection{Eligibility Criteria}

The eligibility criteria for the inclusion of patients in the present study were as follows; 1) histologically proven prostate adenocarcinoma, 2) performance status (Eastern Cooperative Oncology Group) 0 - 2, 3) preserved organ function, 4) no other active malignancy, and 5) written informed consent. Patients who had received prior chemotherapy or radiotherapy, or who were lymph node positive $(\mathrm{N}+)$ and/or metastatic disease (M1) were ineligible. Prior hormone therapy was allowed before enrollment. A CT scan of the abdomen/pelvis and a bone scan were required to rule out metastatic disease. Patients were enrolled only after a thorough evaluation by urologists and radiation oncologists. The study was approved by the Institutional Review Board of the authors' institution. 


\subsection{Daily CBCT as Part of a Rescan Protocol}

Eligible patients with prostate cancer underwent daily CBCT as part of a rescan protocol for large offset to reduce the inter-fractional motion of the prostate using the following protocol: 1) magnesium oxide and dimethylpolysiloxane were administered one week before acquisition of the planning CT scan and were continued until completion of the IMRT to ensure that the patients had regular bowel movements. 2) The patients were instructed to urinate one hour before planning CT scan acquisition and daily irradiation, and, in addition, to drink $200 \mathrm{ml}$ water immediately after urination to ensure that they had an appropriately distended bladder. 3) Daily CBCT images were fused with the planning CT image using the prostate outline, based on the confirmation by one of several radiation oncologists. 4) If large offset, which was defined as prostate motion over the PTV margin plus $>10 \mathrm{~mm}$ or a vanished prostate outline was noted, patients left the treatment couch, and a CBCT rescan and re-registration were performed after the following appropriate countermeasure; if an excessive rectal gas and/or stool were seen, a discharge of the rectal gas was achieved by a cleansing jet of water using a bidet-style toilet and/or an enema, and if full bladder distention was recognized, urination plus consumption of another 200 $\mathrm{ml}$ of water, followed by a one hour wait were carried out. If large offset was still recognized after the re-scan, the appropriate countermeasure(s) and an additional rescan were performed.

\subsection{Treatment Planning and Delivery}

All CBCT images were obtained using a Varian On-Board Imager (OBI, version 1.4, Varian Medical Systems) and approved shifts were analyzed. All patients received definitive IMRT using seven or five fields and daily KV CBCT localization. Patients were irradiated with 76Gy in 38 fractions in the supine position. A CTV was initially defined for each patient as the prostate (and seminal vesicles only for intermediate to high risk cases) as observed on $2.5 \mathrm{~mm}$ thick axial CT images (GE Healthcare; Light Speed RT 16).A planning target volume (PTV) was created by expanding the CTV $4 \mathrm{~mm}$ posteriorly and $7 \mathrm{~mm}$ in all other directions. The rectum was contoured from the inferior border of the anal verge to $1-2 \mathrm{~cm}$ above the plane of the PTV.

The patients were immobilized using a deformable mold before CBCT imaging and treatment. Pelvic skin markings were aligned using laser sights. The CBCT images were next obtained by the gantry rotation around the patient on the couch with a constant speed covering an angle of 364 degrees. One rotation takes about 1 minute. The CBCT was used for all daily imaging. The CBCT field size was set at $35.0 \mathrm{~cm}$ in the LR and AP directions for all patients. The SI field size was set to $16.0 \mathrm{~cm}$. All CBCT scans were performed using one acquisition setting: $125 \mathrm{kV}, 80 \mathrm{~mA}, 13 \mathrm{~ms}$ (standard mode) with a $150 \mathrm{~cm}$ source to imager distance.

Registration for each daily CBCT fused with the treatment planning CT was performed using the prostate outline with an on-board image guidance software system. Manual registration of the images was performed with CBCT imaging by using 3 degrees of freedom (translation only). The corrective shift was then applied to the couch in all axes before irradiation. When using the image guidance software system, this resulted in a lateral, longitudinal, and vertical shift of the treatment planning CT isocenter with respect to the CBCT machine isocenter.

\subsection{Evaluation of the Inter-Fractional Prostate Motion}

The CBCT scans were used to calculate the displacement, which represents the quantification of the mismatch in the prostate contour-based registration between the planning CT and daily CBCT. The displacement of the prostate on CBCT relative to its position on the planning CT after image registration was quantified. The mismatch between the planning CT and each CBCT was calculated in the AP, LR, and SI directions. In the fractions which needed a CBCT rescan, the displacement data after the final rescan were used.

\section{Results}

\subsection{Eligible Patients}

From April 2009 to June 2010, a total of 61 patients were enrolled for this study. The median age of the patients was 65 years (range, 60 - 81 years). The low, intermediate and high risk (NCCN risk) groupshad 5, 26 and 30 patients, respectively. The T stages according to the UICC 6th edition were as follows; T1b in one patient, T1c in 23, T2a in 14, T2b in 4, T2c in 3, T3a in 14 and T3b in 2 patients. The Gleason scores were as follows; score 
2 - 4 in one patient, 5 - 7 in 42 patients and 8 - 10 in 18. The initial PSA level ranged from 5.0 to $118.3 \mathrm{ng} / \mathrm{ml}$ (median, 13.6).

\subsection{Feasibility of Daily CBCT Using the Rescan Protocol}

Forty-eight of 61 patients completed all 38 fractions using daily СВCT on the rescan protocol. In 12 patients, only one of the 38 fractions was irradiated without daily CBCT, because there were issues with the CBCT machine. In the remaining one patient, the continuation of the treatment was refused after 70Gy in 35 fractions. Missing data were recognized for one fraction in one patient. Therefore, a total of 2302 fractions were used for the analysis.

\subsection{CBCT Rescans}

The rescans of CBCT for large offset were performed in 113 (5\%) of 2302 fractions and in 41 (67\%) of 61 patients. The causes for CBCT rescans are listed in Table 1. After the first rescan, the large offset was resolved in 106 (94\%) of 113 fractions (Figure 1). For the remaining 7 fractions, a second rescan was performed in 4 fractions for persistent large offset, three rescans were needed for 2 fractions, and four rescans were used for 1 fraction. The details of the numbers of CBCT rescans per patient are as follows; 0 in 20 (33\%) patients, 1 - 3 in 29 (48\%) patients, 4 - 7 in $10(16 \%)$ patients, and 8 - 10 in 2 (3\%) patients. Figure 2 shows the distribution of the CBCT rescans during all 38 fractions of IMRT.

\subsection{Inter-Fractional Motion of CBCT on the Rescan Protocol}

The total mean and SD of the inter-fractional prostate motion in the AP, LR, and SI directions were $1.1 \pm 2.4$, $-0.1 \pm 2.3$, and $0.7 \pm 3.0 \mathrm{~mm}$, respectively (Figure 3). The rate of the corrective shift within the PTV margin (the CTV plus $4 \mathrm{~mm}$ posterior and $7 \mathrm{~mm}$ in all other directions) was 97.4, 99.9, and 97.6\% in the AP, LR, and SI directions, respectively (Table 2). In 115 (5\%) of the 2302 CBCT fractions, a corrective shift over the PTV margin occurred. The rate of $<5 \mathrm{~mm}$ inter-fraction prostate motion in the AP, LR, and SI directions was 91.7, 95.1, and $86.0 \%$, respectively (Table 3 ).

Table 1. Cause of the large offset noted during the CBCT rescans.

\begin{tabular}{cc}
\hline Variable & $\mathrm{n}=113(\%)$ \\
\hline Excess rectal gas & $94(83)$ \\
Artifact of the CBCT & $14(12)$ \\
Excess feces & $3(3)$ \\
Human error & $2(2)$ \\
\hline
\end{tabular}

Table 2. The prostate inter-fractional motion over the PTV margin.

\begin{tabular}{|c|c|c|c|}
\hline \multirow{2}{*}{ Inter-fractional motion over the PTV margin (mm) } & \multicolumn{3}{|c|}{ Fractions $(\mathrm{n}=2302)(\%)$} \\
\hline & AP & LR & SI \\
\hline$<1.0$ & $2242(97.4)$ & 2300 (99.9) & $2246(97.6)$ \\
\hline $1.0-1.9$ & $27(1.1)$ & $2(0.1)$ & $24(1.0)$ \\
\hline $2.0-2.9$ & $18(0.8)$ & 0 & $18(0.8)$ \\
\hline $3.0-3.9$ & $9(0.4)$ & 0 & $6(0.3)$ \\
\hline $4.0-4.9$ & $3(0.1)$ & 0 & $3(0.1)$ \\
\hline$\geq 5.0$ & $3(0.1)$ & 0 & $5(0.2)$ \\
\hline
\end{tabular}

Abbreviations: PTV = planning target volume; AP = anterior-posterior; LR = left-right; SI = superior-inferior. 
Table 3. Previous studies of the daily inter-fractional motions of the prostate measured by various image guidance methods.

\begin{tabular}{|c|c|c|c|c|c|}
\hline \multirow{2}{*}{ Study ${ }^{\text {ref.) }}$} & \multirow{2}{*}{ Patients (n) } & \multirow{2}{*}{ Scans (n) } & \multicolumn{3}{|c|}{ Rate of $<5 \mathrm{~mm}$ inter-fractional motion (\%) } \\
\hline & & & AP & LR & SI \\
\hline Ultrasound $^{16)}$ & 40 & 1019 & 61.4 & 69.3 & 59.6 \\
\hline Fiducial seed marker ${ }^{16)}$ & 40 & 1019 & 64.5 & 78.4 & 68.4 \\
\hline CT on rails ${ }^{17)}$ & 20 & 1870 & 58.0 & 78.0 & 83.0 \\
\hline MV CBCT ${ }^{15)}$ & 24 & 793 & 64.5 & 87.0 & 93.1 \\
\hline Our study & 61 & 2302 & 91.7 & 95.1 & 86.0 \\
\hline
\end{tabular}

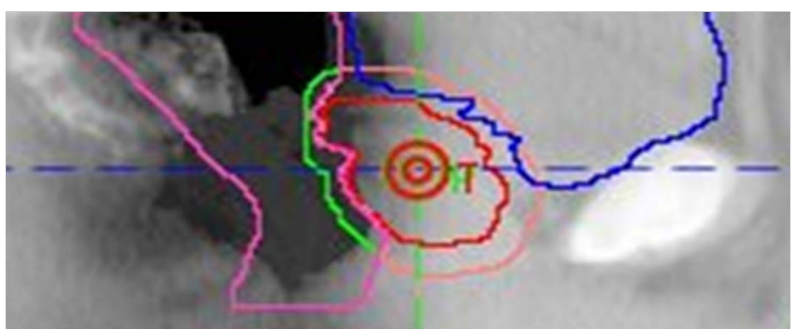

(a)

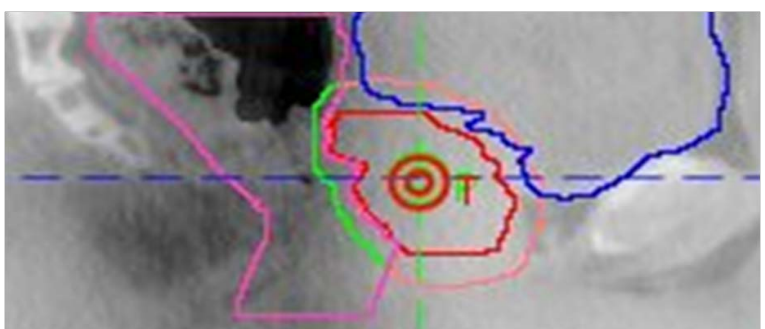

(b)

Figure 1. A case with severe misregistration caused by excess rectal gas. The red line is the CTV, pink line is PTV, the purple line is the rectum and the blue line is the bladder on the planning CT. (a) Severe misregistration with excess rectal gas is shown in a fused image consisting of the planning CT and initial CBCT image. (b) Severe misregistration was resolved after the discharge of the rectal gas as shown in a fused image consisting of the planning CT and rescan CBCT image.

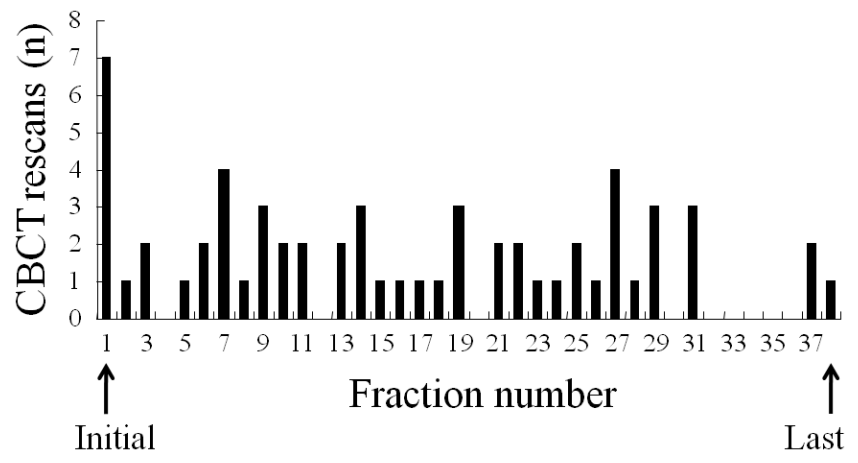

Figure 2. The distribution of the CBCT rescans during all 38 fractions of IMRT.

\section{Discussion}

The present study is the first to prospectively assess the daily CBCT as part of a rescan protocol to reduce the inter-fractional motions of the prostate. In addition, to the best our knowledge, the current study is one of the largest series of patients with prostate cancer treated with daily IGRT. The issue of prostate motion has been investigated by many research groups. The previously reported range of inter-fractional motion of the prostate, as measured by a standard deviation from the initial setup, was $0.7-5.2 \mathrm{~mm}$ for all directions [2] [3] [8]-[13]. Table 3 lists the previous studies of the daily inter-fractional motions of the prostate measured by various image guidance methods [5]-[7]. Bylund et al. reported a total of 984 daily megavoltage CBCT images from 24 patients undergoing definitive IMRT for localized prostate cancer; the mean displacement of the inter-fractional motion was 4.8, 2.9, and $2.1 \mathrm{~mm}$ in the AP, LR, and SI directions, respectively [5]. Scarbrough et al. investigated the inter-fractional motions of the prostate in 40 patients using ultrasound and fiducial seed markers; with ultrasound, the rates of $<5 \mathrm{~mm}$ inter-fractional motion were $61.4 \%, 69.3 \%$, and $59.6 \%$ in the AP, LR, SI directions respectively, and, with fiducial seed markers, were $64.5 \%, 78.4 \%$, and $68.4 \%$ in the AP, LR, SI directions, 


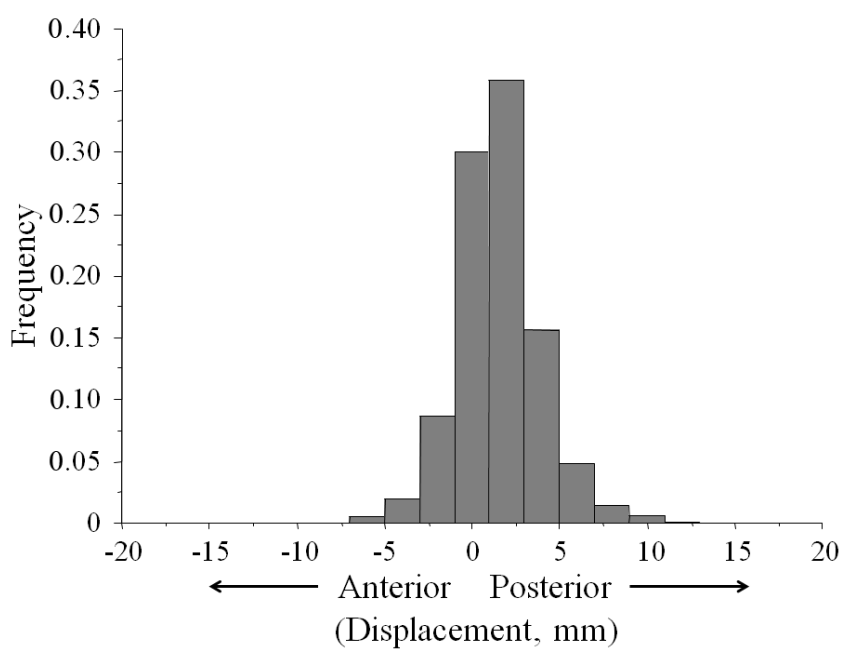

(a)

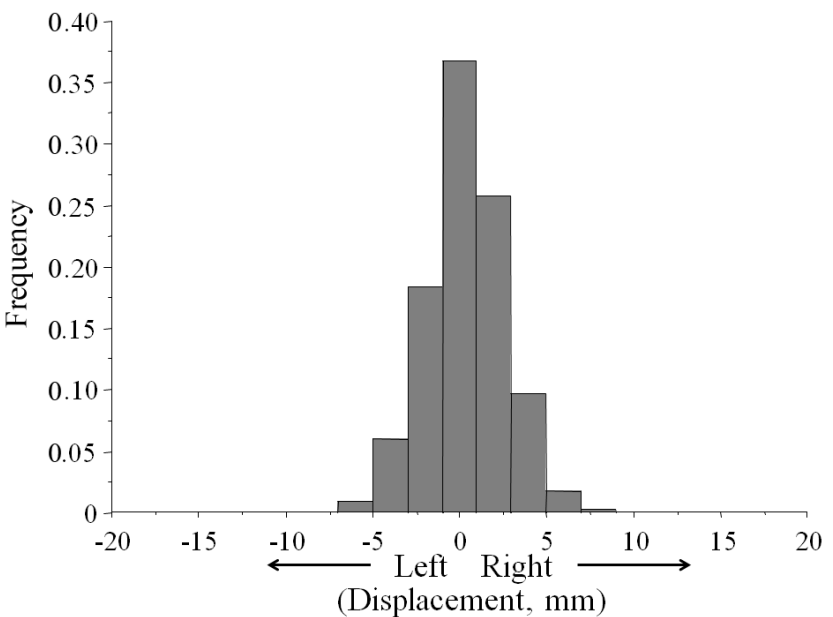

(b)

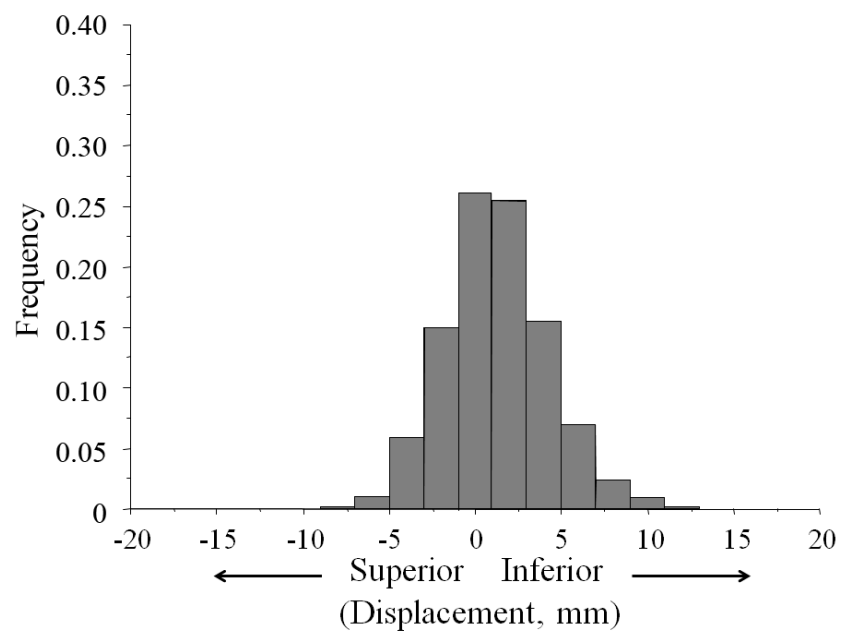

(c)

Figure 3. The frequency distributions for the daily inter-fractional prostate displacement when using the daily CBCT as part of a rescan protocol in the AP (a), LR (b), and SI (c) directions. 
respectively [6]. In the current prospective study of daily KV CBCT as part of a rescan protocol, both the mean displacement of the inter-fractional motion and the rate of $<5 \mathrm{~mm}$ inter-fractional motion of the prostate were better than those in the previous studies (Table 3), thus indicating this protocol to be promising for improving radiation therapy.

The general consensus among the studies of the inter-fractional motion of the prostate was that rectal filling has a larger effect on prostate motion than bladder filling [2] [3] [8]-[13]. Antolak et al. reported in CT studies during a course of RT that the prostate mobility was not significantly correlated with the bladder volume, however, the mobility of both the prostate and seminal vesicles significantly correlated with the rectal volume [9]. There have been several reports on the use of an endorectal balloon tube to reduce rectal gas filling, which immobilizes the prostate gland and pushes the prostate toward the pubic bone, thus resulting in rectal wall sparing and reduced rectal toxicity [13] [14]. Wachter et al. reported a large experience involving the use of an endorectal balloon tube for daily prostate treatment, and maximal displacements of the posterior prostate border $(>5 \mathrm{~mm})$ were found in $8 / 10$ patients without a rectal balloon and in only 2/10 patients with the balloon [14]. However, van Lin et al. reported a clinical study of 22 patients irradiated with an endorectal balloon tube in situ and in 30 patients without an endorectal balloon tube, and the investigated endorectal balloon tube did not reduce the inter-fractional prostate motion [15]. Our prospective data showed that the large offset was mainly caused by excess rectal gas, and a rescan of CBCT and re-registration were performed after appropriate countermeasures were taken, such as a discharge of the rectal gas. Although the rescan was needed in 5\% of the IMRT fractions, it could successfully resolve the large offset mainly caused by excess rectal gas. Therefore, daily CBCT may be useful, not only to correct for setup uncertainty, but also to examine rectal filling, and daily CBCT as part of a rescan protocol is promising to reduce the inter-fractional motion of the prostate.

With the increased use of CBCT for daily patient setup, the accumulated dose from CBCT may be significantly higher than that from simulation CT or portal imaging, and these additional doses may present a biological risk to radiosensitive organs [16] [17]. For example, Ding et al. reported the detailed information concerning the additional imaging doses to patients' radiosensitive organs from a KV CBCT scan; for an abdominal scan, the mean doses were 3 and 7 cGy to the prostate and 7 and 17 cGy to the femoral heads for a large adult patient and a 31-month-old pediatric patient, respectively [17]. Recently, a method to incorporate the daily megavoltage CBCT dose into the IMRT planning process was developed and implemented in the treatment of prostate cancer patients [18]. This technique has the potential to greatly improve the accuracy of treatment delivery, while maintaining target coverage and sparing the organs at risk. Although the current study of the daily KV CBCT as part of a rescan protocol demonstrated a promising decrease in the inter-fractional motion of the prostate, further investigations of IMRT planning incorporating the imaging doses from KV IGRT may be needed.

There were several limitations associated with the current study. The corrective shift required could not be scored when large offset was recognized, because the overall outline of the prostate was not identified in most cases, and the prostate outline could not be fused exactly with the planning CT image in such cases. Therefore, in the fractions which needed the CBCT rescan for large offset, only the displacement data after the final rescan were available and thus were analyzed.

\section{Conclusion}

In summary, large offset was recognized in $5 \%$ of all fractions and in $67 \%$ of all patients in the current prospective study using daily KV CBCT as part of a rescan protocol. However, the rescan protocol could successfully resolve the large offset, which was mainly caused by excessive amounts of rectal gas. The inter-fractional motions of daily KV CBCT as part of a rescan protocol were better than those in the previously reported studies. Daily CBCT may therefore be useful to correct for setup uncertainty and to examine rectal filling, and daily $\mathrm{CBCT}$ as part of a rescan protocol is a promising modality to reduce the inter-fractional motion of the prostate.

\section{References}

[1] Zelefsky, M.J., Chan, H., Hunt, M., Yamada, Y., Shippy, A.M. and Amols, H. (2006) Long-Term Outcome of High Dose Intensity Modulated Radiation Therapy for Patients with Clinically Localized Prostate Cancer. The Journal of Urology, 176, 1415-1419. http://dx.doi.org/10.1016/j.juro.2006.06.002

[2] Roeske, J.C., Forman, J.D., Mesina, C.F., He, T., Pelizzari, C.A., Fontenla, E., Vijayakumar, S. and Chen, G.T. (1995) Evaluation of Changes in the Size and Location of the Prostate, Seminal Vesicles, Bladder, and Rectum during a 
Course of External Beam Radiation Therapy. International Journal of Radiation Oncology*Biology*Physics, 33, 13211329. http://dx.doi.org/10.1016/0360-3016(95)00225-1

[3] Van Herk, M., Bruce, A., Kroes, A.P., Shouman, T., Touw, A. and Lebesque, J.V. (1995) Quantification of Organ Motion during Conformal Radiotherapy of the Prostate by Three Dimensional Image Registration. International Journal of Radiation Oncology*Biology*Physics, 33, 1311-1320. http://dx.doi.org/10.1016/0360-3016(95)00116-6

[4] Huang, E., Dong, L., Chandra, A., Kuban, D. A., Rosen, I.I., Evans, A. and Pollack, A. (2002) Intrafraction Prostate Motion during IMRT for Prostate Cancer. International Journal of Radiation Oncology*Biology*Physics, 53, 261-268. http://dx.doi.org/10.1016/S0360-3016(02)02738-4

[5] Bylund, K.C., Bayouth, J.E., Smith, M.C., Hass, A.C., Bhatia, S.K. and Buatti, J.M. (2008) Analysis of Interfraction Prostate Motion Using Megavoltage Cone Beam Computed Tomography. International Journal of Radiation Oncology*Biology*Physics, 72, 949-956. http://dx.doi.org/10.1016/j.ijrobp.2008.07.002

[6] Scarbrough, T.J., Golden, N.M., Ting, J.Y., Fuller, C.D., Wong, A., Kupelian, P.A. and Thomas Jr., C.R. (2006) Comparison of Ultrasound and Implanted Seed Marker Prostate Localization Methods: Implications for Image-Guided Radiotherapy. International Journal of Radiation Oncology*Biology*Physics, 65, 378-387.

http://dx.doi.org/10.1016/j.ijrobp.2006.01.008

[7] Dong, L., de Crevoisier, R., Bonnen, M., Lee, A., Cheung, R., Wang, H., O’Daniel, J., Mohan, R., Cox, J. and Kuban, D. (2004) Evaluation of an Ultrasound-Based Prostate Target Localization Technique with an In-Room CT-on-Rails. International Journal of Radiation Oncology*Biology*Physics, 60, S332-S333. http://dx.doi.org/10.1016/j.ijrobp.2004.07.155

[8] Melian, E., Mageras, G.S., Fuks, Z., Leibel, S.A., Niehaus, A., Lorant, H., Zelefsky, M., Baldwin, B. and Kutcher, G.J. (1997) Variation in Prostate Position Quantitation and Implications for Three-Dimensional Conformal Treatment Planning. International Journal of Radiation Oncology*Biology*Physics, 38, 73-81. http://dx.doi.org/10.1016/S0360-3016(97)00221-6

[9] Antolak, J.A., Rosen, I.I., Childress, C.H., Zagars, G.K. and Pollack, A. (1998) Prostate Target Volume Variations during a Course of Radiotherapy. International Journal of Radiation Oncology*Biology*Physics, 42, 661-672. http://dx.doi.org/10.1016/S0360-3016(98)00248-X

[10] Althof, V.G.M., Hoekstra, C.J.M. and te Loo, H.-J. (1996) Variation in Prostate Position Relative to Adjacent Bony Anatomy. International Journal of Radiation Oncology*Biology*Physics, 34, 709-715. http://dx.doi.org/10.1016/0360-3016(95)02162-0

[11] Balter, J.M., Sandler, H.M., Lam, K., Bree, R.L., Lichter, A.S. and ten Haken, R.K. (1995) Measurement of Prostate Movement over the Course of Routine Radiotherapy Using Implanted Markers. International Journal of Radiation Oncology*Biology*Physics, 31, 113-118. http://dx.doi.org/10.1016/0360-3016(94)00382-U

[12] Crook, J.M., Raymond, Y., Salhani, D., Yang, H. and Esche, B. (1995) Prostate Motion during Standard Radiotherapy as Assessed by Fiducial Markers. Radiotherapy and Oncology, 37, 35-42. http://dx.doi.org/10.1016/0167-8140(95)01613-L

[13] Vigneault, E., Pouliot, J., Laverdiere, J., Roy, J. and Dorion, M. (1997) Electronic Portal Imaging Device Detection of Radioopaque Markers for the Evaluation of Prostate Position during Megavoltage Irradiation: A Clinical Study. International Journal of Radiation Oncology*Biology*Physics, 37, 205-212. http://dx.doi.org/10.1016/S0360-3016(96)00341-0

[14] Wachter, S., Gerstner, N., Dorner, D., Goldner, G., Colotto, A., Wambersie, A. and Potter, R. (2002) The Influence of a Rectal Balloon Tube as Internal Immobilization Device on Variations of Volumes and Dose-Volume Histograms during Treatment Course of Conformal Radiotherapy for Prostate Cancer. International Journal of Radiation Oncology*Biology*Physics, 52, 91-100. http://dx.doi.org/10.1016/S0360-3016(01)01821-1

[15] van Lin, E.N., van der Vight, L.P., Witjes, J.A., Huisman, H.J., Leer, J.W. and Visser, A.G. (2005) The Effect of an Endorectal Balloon and Off-Line Correction on the Interfraction Systematic and Random Prostate Position Variations: A Comparative Study. International Journal of Radiation Oncology*Biology*Physics, 61, 278-288. http://dx.doi.org/10.1016/j.ijrobp.2004.09.042

[16] Wen, N., Guan, H., Hammoud, R., Pradhan, D., Nurushev, T., Li, S. and Movsas, B. (2007) Dose Delivered from Varian’s CBCT to Patients Receiving IMRT for Prostate Cancer. Physics in Medicine and Biology, 52, 2267-2276. http://dx.doi.org/10.1088/0031-9155/52/8/015

[17] Ding, G.X. and Coffey, C.W. (2009) Radiation Dose from Kilovoltage Cone Beam Computed Tomography in an Image-Guided Radiotherapy Procedure. International Journal of Radiation Oncology*Biology*Physics, 73, 610-617. http://dx.doi.org/10.1016/j.ijrobp.2008.10.006

[18] Miften, M., Gayou, O., Reitz, B., Fuhrer, R., Leicher, B. and Parda, D.S. (2007) IMRT Planning and Delivery Incorporating Daily Dose from Mega-Voltage Cone-Beam Computed Tomography Imaging. Medical Physics, 34, 37603767. http://dx.doi.org/10.1118/1.2779127 
Scientific Research Publishing (SCIRP) is one of the largest Open Access journal publishers. It is currently publishing more than 200 open access, online, peer-reviewed journals covering a wide range of academic disciplines. SCIRP serves the worldwide academic communities and contributes to the progress and application of science with its publication.

Other selected journals from SCIRP are listed as below. Submit your manuscript to us via either submit@scirp.org or Online Submission Portal.
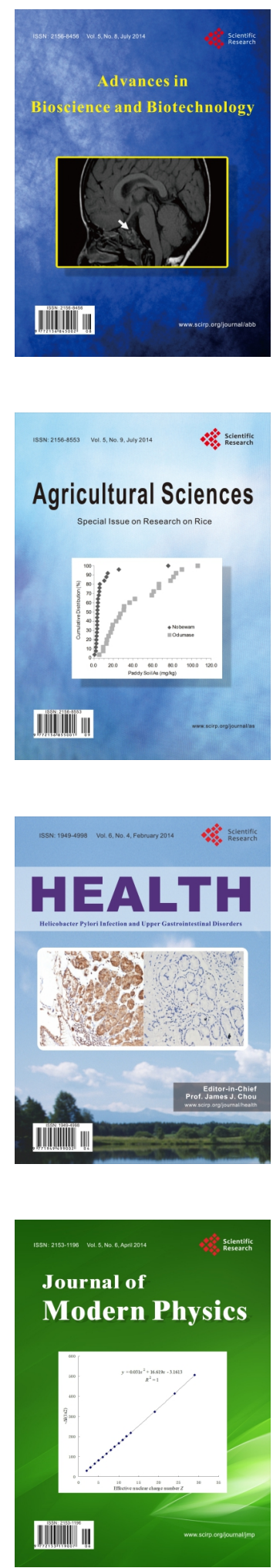
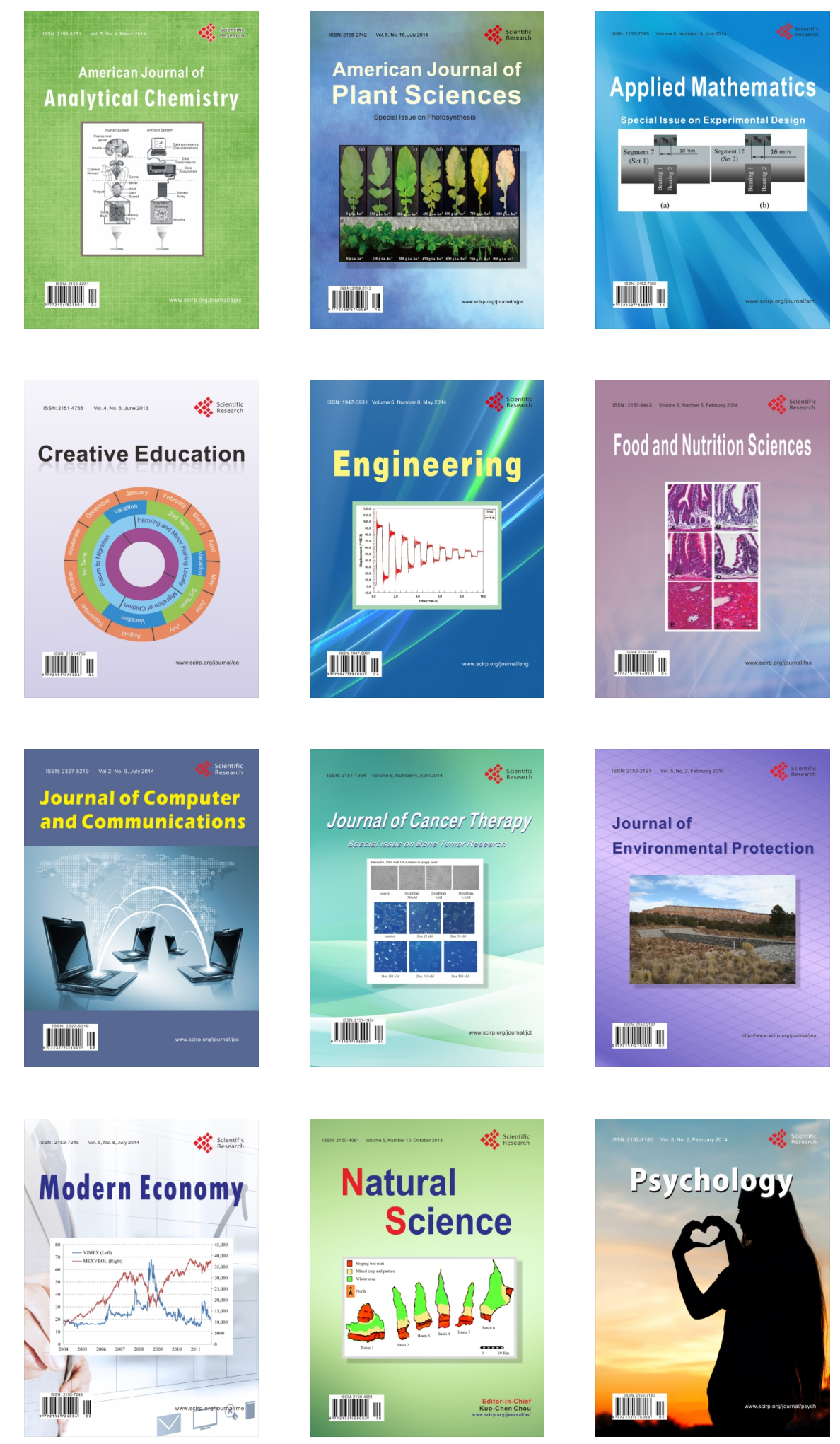